Among police registrants at Survey Wave 2, 5-6 years after 9/11/ 01, four unique trajectories of probable PTSD symptoms were identified: resilient (83\%), recovered $(2.5 \%)$, delayed onset (11.2\%) and chronic (5.3\%). The current study documents the longitudinal trajectories of PTSD in police officers exposed to the WTC attacks at Wave 3 (2011-2012), over 10 years since the WTC attack.

Method We examined the prevalence of probable PTSD at the Wave 3 survey using a cut-off score of 44 or greater on the event-specific Posttraumatic Stress Disorder Checklist (PCL) and at least one re-experiencing symptom (DSM-IV criterion B), three avoidance or numbing symptoms (DSM-IV criterion C), and two hyperarousal symptoms (DSM-IV criterion D).

Results Probable PTSD was $14.4 \%$ (95\% CI 13.0-15.9\%) at Wave 2 and 12.9\% (95\% CI, 11.6-14.3\%) at Wave 3. Significant predictors of chronic probable PTSD 10 years post-disaster $(\mathrm{n}=$ $59 / 2241,2.6 \%$ ) include age 45-69 (aOR 3.16, 95\% CI, 1.76.0), number of stressful events witnessed on 9/11/01 (aOR $3.00,1.6-5.8$ ), five or more stressful life events since 9/11/01 (aOR 5.42, 1.9-15.2), and unmet mental health care needs (aOR $6.86,3.3-14.1$ ). Protective factors include social support (aOR $0.34,0.1-0.97)$ and number of close friends or relatives (aOR $0.92,0.87-0.98)$.

Conclusions Chronic probable PTSD among police responders continues to be a significant problem, associated both with intervening stressful life events and unmet mental health care needs.

\section{SHIFT-WORK AND CANCER RESEARCH: DEVELOPING A QUANTITATIVE APPROACH TO ASSESS CHRONODISRUPTION}

'Thomas C Erren, 'J Valérie Gross, ${ }^{2}$ Peter Morfeld. 'University of Cologne, Cologne, Germany; ${ }^{2}$ Evonik Industries, Essen, Germany

\subsection{6/oemed-2014-102362.173}

Objectives In 2007, IARC classified shift-work involving "circadian disruption" as probably carcinogenic [Group 2A]. Thereafter, 23 shift-work studies into a series of malignant endpoints provided additional epidemiologic evidence regarding chains of cancer causation, which appear biologically plausible when experimental data are considered. Since none of the studies specifically assessed "circadian disruption" or disturbed chronobiology, we ask the following: Does published research suffer from chronobiological errors? How could we assess circadian or chronodisruption $[\mathrm{CD}]$ in future epidemiological studies?

Method On the basis of chronobiological insights we develop a quantitative approach to assess CD for different chronotypes and for different shift-work regimens. We examine how chronobiological errors may have affected studies published so far with a focus on those which considered chronobiological information but were confined to night-work.

Results We illustrate that ignoring "internal time", which is critically determined by an individual's chronotype, may lead to what we propose to call "IT errors". In addition, assessing biological effects associated with shift-work should also include associated activities and therefore temporal information beyond the nominal shift time. Such errors of truncating relevant "external time" information (activities at chronobiologically unusual times start before and do not end with the shift) we propose to coin "ET errors". We propose methodologically how observational research may avoid potential chronobiological biases and how chronodisruption can be computed.
Conclusions Avoiding both IT and ET errors with regard to any - and not just night - shift work may be a prerequisite to assess causal links between shift-work and cancer which may remain otherwise masked.

\section{Poster presentation}

\section{COMPREHENSIVE ANALYSIS OF RESEARCH AND PROGRAM-BASED STUDIES ON OCCUPATIONAL HEALTH AND SAFETY IN THE PHILIPPINES}

Jinky Leilanie Lu. National Institutes of Health, Univeristy of the Philippines, Manila, The Philippines

\subsection{6/oemed-2014-102362.174}

Objectives This study looked into the state of occupational health and safety in the country. Specifically, the objectives were 1) to show the current condition of workers, both local and migrant, in terms of their workplace condition and hazard exposures; and 2) to present occupational diseases and illnesses in various industries and occupational groupings in the Philippines. Method The methodology consisted of comprehensive analysis of records and statistics on occupational safety and health, and related variables from various institutions. Data were gathered from reviews of literatures, related research studies, and documentary research at the Occupational Safety and Health Centre. Analysis of data was done through a critical appraisal of the current status of occupational and health safety in the Philippines in terms of occupational diseases, injuries, and accidents, and existing occupational health and safety policies.

Results The study showed occupational hazards and health and safety conditions in various industries, occupational settings, and job groupings such as in the industrial sector, manufacturing, mining, agriculture, fishing, and cement manufacturing. It also looked into small scale and informal industries such as tanning, laundry shops, pyrotechnique manufacturing and the like. Special segments of the labour force including the women workers, child labourers and migrant workers were also covered. In all these sectors and industries, the study showed attendant occupational diseases and injuries arising from occupational hazards.

Conclusions The study showed more complete data on occupational health and safety in the Philippines considering that there is insufficient collection of OHS data by concerned government agencies.

\section{EFFECTS OF AGRICULTURAL WORK PRACTICES AND PESTICIDE USE ON OCCUPATIONAL HEALTH OF FARMERS}

Jinky Leilanie Lu. National Institutes of Health, Univeristy of the Philippines Mnaila, Anila, The Philippines

\subsection{6/oemed-2014-102362.175}

Objectives This study aimed to identify the associated health symptoms between high and low exposed groups to pesticide, and to come up with an intervention pesticide program for our farmers in the vegetable industry.

Method Survey questionnaires were used to look into pesticide exposures and work practices of 534 farmers in the largest eggplant producing province in the northern Philippines. Physical health assessment was conducted by medical doctors to look into the 
health status of farmers who ahve been using pesticides for the past years. Laboratory examination of blood was also done, including blood cholinesterase to dertermine organophosphate exposures.

Results Majority were males (53.4\%), married (80.5\%) with a mean age of 47 years old. The most commonly used pesticides were Tamaron (36.1\%), Dithane (34.1\%), Sumicidine (29.0\%), Selecron (24.9\%) and Lannate (15.2\%). Tamaron, being the most commonly used, has an active ingredient of methamidophos and classified as an organophosphate pesticide. Meanwhile, Dithane, a mancozeb, is a dithiocarbamate pesticide and Sumicidine is a pyrethroid with an active ingredient of fenvalerate. In addition, Selecron is composed mainly of ingredients of prochloroz $\mathrm{mn}$ and spinosad, respectively (Table 2). During their agricultural work, farmers used pesticides mainly for three to six hours per day $(51.2 \%)$, one to two days weekly $(86.8 \%)$ and three to four weeks per month (82.3\%). 40.9\% who underwent the physical examination were diagnosed to have abnormal assessment results. Analyses indicated that pesticide use and risk factors were found to have association at $p=0.05$ with easy fatigability, weight loss, loss of appetite, cerebellar function, creatinine levels, haemoglobin, mean corpuscular volume, mean corpuscular haemoglobin count, and platelet count. 5.3\% of the farmers were found to have abnormal platelet count. Platelet count may indicate whether the patient is having bleeding problems, infectious processes, bone marrow depression, malignancies or anaemia. For RBC cholinesterase activity, it was found that $50.8 \%$ of the samples showed depression in activity.

Conclusions This study has demonstrated the interaction between the farmers' pesticide exposure and work practices, as well as physical, neurological and laboratory assessment findings. The study shows that farmers are at a considerable risk of either acute or chronic pesticide poisoning because of improper work practices. Around $40.9 \%$ of the farmers were diagnosed to have abnormal physical examination findings while $4.6 \%$ presented problems in their neurological functioning and less than $10 \%$ of the farmers exhibited abnormal laboratory results. The author recommends the use of the information gathered in this study to improve current policies and standards with regards to surveillance of pesticide use.

\section{TRENDS OF OCCUPATIONAL INJURY IN THE PHILIPPINES: IMPLICATIONS FOR POLICY}

Jinky Leilanie Lu. National Institutes of Health, Univeristy of the Philippines Manila, Manila, The Philippines

\subsection{6/oemed-2014-102362.176}

Objectives This study aimed to review and assess the prevalence and incidence of occupational injuries in the Philippines.

Method Data collection were done from various agencies, namely, Bureau of Labour and Employment Statistics (BLES) of the Department of Labour and Employment (DOLE), Labour Force Survey of National Statistics Office, among others. Hospital-based surveys and newspaper reports were also sources of data for this study.

Results The review showed that about 358000 fatal and 337 million non-fatal occupational accidents in the world, and 1.95 million deaths from work-related diseases. Occupational injuries in the Philippines showed 22265 cases in 2003, and 47235 cases in 2007. The manufacturing industries registered the highest number of cases Out of the reported cases of occupational injuries, 178 resulted in death in 2000, and 116 deaths in 2007. Injury occurred at 6 injury cases per 500 full-time workers, or 1 injury case for every 88 workers in 2000. In the following years, it declined to 4 cases per full-time worker in 2003, and 3 cases for every 88 workers in 2007. Based on hospital records, there was a total of 9521 injury cases reported for the first quarter of 2010 in 77 government and private hospitals in the country. The cause of injury mostly occurred on the road (44.4\%), and workrelated injuries were reported at $7.8 \%$.

Conclusions The review of occupational injuries in the Philippines showed major trends in injuries, causes of injuries and rates and severity of injury. However, the data lack more specific and segregated information per industry and occupational grouping, as well as identification of risk factors associated with these injuries. Therefore, improvements in injury surveillance and documentation of injury cases as well as research into risk factors at work should be done. All these efforts should lend towards prevention strategies and guidelines on occupational injuries in the Philippines. Also, there is a need to have a standard nomenclature of occupational injuries, starting from the primary data sources (company clinics) which are the bases of the national data. It is suggested that data collection on occupational injuries be a national scale, and not merely randomised collection of data for small, medium and large industries.

\section{A NEW MODEL FOR OUTREACH TO SMALL INDUSTRIAL FIRMS: SAFETY AUDIT RESULTS FROM THE NATIONAL MACHINE GUARDING PROGRAM}

${ }^{1}$ Samuel Yamin, ${ }^{1}$ David Parker, ${ }^{2}$ Lisa Brosseau, ${ }^{1}$ Min Xi, ${ }^{3}$ Bob Gordon. ${ }^{1}$ Park Nicollet Institute, St. Louis Park, MN, USA; ${ }^{2}$ University of Illinois at Chicago, Chicago, IL, USA; ${ }^{3}$ Interrobang Group, Minneapolis, MN, USA

\subsection{6/oemed-2014-102362.177}

Objectives Small industrial firms frequently lack occupational safety and health (OSH) expertise. In addition, development of effective, widely applicable OSH interventions for small businesses has proven to be a persistent challenge. The National Machine Guarding Program is an intervention currently underway to address these issues among small (3-150 employees) metal fabrication businesses.

Method A partnership was formed with workers' compensation insurers to recruit businesses for an intervention designed to prevent machine-related injuries and improve safety programs such as lockout/tagout. Participants receive a baseline evaluation, two intervention visits, and a follow-up evaluation. Pooled results from baseline safety assessments will be presented.

Results 221 businesses from 29 U.S. states were enrolled. At baseline, the mean score for machine safety audit was $73.7 \%$, however, only $67.5 \%$ of items concerning point of operation safeguards were present on average. For shop-wide safety programs and policies, mean audit score was $43.4 \%$. Safety program/policy scores were significantly lower among smaller businesses, whereas machine safeguarding equipment scores were similar across all size strata.

Conclusions The National Machine Guarding Program represents a new intervention model for widespread outreach to small industrial firms. Baseline safety audit results show a need for improvement in shop-wide safety programs and in critical areas of machine safeguarding. Preliminary results indicate that this is an effective model for recruiting small metal fabrication businesses into a nationwide intervention. Effectiveness of intervention programs in improving these aspects of injury prevention will be evaluated to determine whether broader application is warranted. 